\title{
Prevalence of methicillin-resistant Staphylococcus aureus skin and nasal carriage isolates from bovines and its antibiogram
}

\author{
Alok Kumar ${ }^{1}$, Purushottam Kaushik ${ }^{1}$, Anjay ${ }^{1}$, Pankaj Kumar ${ }^{2}$ and Manoj Kumar ${ }^{2}$ \\ 1. Department of Veterinary Public Health \& Epidemiology, Bihar Veterinary College, Patna, Bihar, India; 2. Department \\ of Veterinary Microbiology, Bihar Veterinary College, Patna, Bihar, India. \\ Corresponding author: Purushottam Kaushik, e-mail: drkaushikvet@gmail.com, \\ Co-authors: AK: vetalok52@gmail.com, Anjay: dranjayvet@gmail.com,PK: pankajimmunol@gmail.com, \\ MK: drmanojmicro@rediffmail.com \\ Received: 26-11-2016, Accepted: 15-04-2017, Published online: 04-06-2017
}

doi: 10.14202/vetworld.2017.593-597 How to cite this article: Kumar A, Kaushik P, Anjay, Kumar P, Kumar M (2017) Prevalence of methicillin-resistant Staphylococcus aureus skin and nasal carriage isolates from bovines and its antibiogram, Veterinary World, 10(6): 593-597.

\begin{abstract}
Aim: This study was conducted to determine the prevalence of methicillin-resistant Staphylococcus aureus (MRSA) in cattle and buffalo and to study their antibiotic resistance pattern.

Materials and Methods: A total of 136 samples (skin and nasal swab) from cattle and buffalo were collected. MRSA was identified by conventional bacterial culture techniques which were further confirmed by amplification of $S$. aureus-specific 16S rRNA by polymerase chain reaction (PCR). The isolates were further analyzed for the presence of mecA gene by PCR. The antimicrobial susceptibility profiling was performed by disc diffusion method.

Results: The prevalence of MRSA in the current study was $28.57 \%$ and $34.28 \%$ in cattle nasal and skin swab, respectively, with an overall prevalence of $31.43 \%$ MRSA among cattle. Buffalo nasal and skin sample showed MRSA prevalence of $54.55 \%$ and $39.4 \%$, respectively, with $46.9 \%$ overall prevalence. PCR could detect mecA gene in $36.4 \%$ and $58 \%$ MRSA isolates from cattle and buffalo, respectively. Antimicrobial susceptibility test found MRSA resistant to penicillin and oxytetracycline (88\% each), cefoxitin $(75 \%)$, cotrimoxazole $(62 \%)$, and amoxyclav $(50 \%) .100 \%$ sensitivity was observed against ciprofloxacin, amikacin, chloramphenicol, and gentamicin. Three (16.7\%) MRSA isolates from buffalo were found resistant to vancomycin.
\end{abstract}

Conclusion: Cattle and buffalo were identified as a potential carrier of MRSA in Bihar (India). The isolation of vancomycinresistant $S$. aureus (VRSA) in the current study indicates the emergence of VRSA in animal population which may be transmitted to the human beings working in close contact to the animals.

Keywords: antibiogram, bovine, mecA gene, methicillin resistant Staphylococcus aureus.

\section{Introduction}

Staphylococcus aureus is an opportunistic pathogen of human and animal [1] which usually colonizes anterior nares and causes infection in immunocompromised patients [2]. Alexander Ogston in 1880 first isolated $S$. aureus and first isolation of penicillin-resistant S. aureus was made in 1942 from clinical cases [3]. It was later on circumvented by the introduction of methicillin and the first methicillin resistant $S$. aureus (MRSA) appeared in the year 1961 which later on became a serious nosocomial infection worldwide [4]. The organism has developed resistance to methicillin by integration of a 21-67 kbp mobile genetic element, termed as staphylococcal cassette chromosome mec [5] into their genome which harbors the methicillin resistance (mecA) gene and other antibiotic resistance determinants $[6,7]$, leaving vancomycin as

Copyright: Kumar, et al. Open Access. This article is distributed under the terms of the Creative Commons Attribution 4.0 International License (http://creativecommons.org/licenses/ by/4.0/), which permits unrestricted use, distribution, and reproduction in any medium, provided you give appropriate credit to the original author(s) and the source, provide a link to the Creative Commons license, and indicate if changes were made. The Creative Commons Public Domain Dedication waiver (http:// creativecommons.org/publicdomain/zero/1.0/) applies to the data made available in this article, unless otherwise stated. the drug of last choice to treat the MRSA infections. This gene encodes for a penicillin-binding protein 2 (PBP2a) expressed in the bacterial cell wall and has a low affinity for $\beta$-lactam antibiotics. Thus, this group of antibiotics becomes insensitive to bacteria expressing mecA gene.

Data regarding the prevalence of MRSA in India have been reported from various regions [8-11]. However, no systematic study is available on the prevalence and antimicrobial resistance pattern of MRSA, in Bihar.

Hence, this study was planned for isolation, identification and molecular detection of methicillin resistance in staphylococci and their antibiotic resistance pattern from bovines.

\section{Materials and Methods}

\section{Collection of sample}

Skin and nasal swab samples (136) from apparently healthy cattle and buffalo were collected from Institutional Livestock Farm Complex, Bihar Veterinary College (BVC), Patna, and from the patients coming to teaching veterinary clinical complex of BVC, Patna. The sample comprised skin and anterior nostril swab of cattle (35 each) and buffalo 
(33 each). The skin samples were collected from the axillary region of the animals. The samples were inoculated in tryptone soya broth (TSB) with $10 \%$ sodium chloride salt (TSB-S) (Hi-media) and incubated at $37^{\circ} \mathrm{C}$ for $24 \mathrm{~h}$. The samples which showed turbidity in TSB-S broth were streaked on mannitol salt agar (Hi-media) with $6 \mathrm{mg} / \mathrm{L}$ oxacillin (O-MS agar) and incubated for $24 \mathrm{~h}$ at $37^{\circ} \mathrm{C}$. Bacterial growth with mannitol fermentation was observed for the presence of round-shaped, typical golden, yellow, or pale color colonies of oxacillin-resistant staphylococci. The presumptive isolates were further confirmed by Gram'sstaining (Hi-media) and catalase test [12]. The test was performed with a loopful culture of isolates mixed with a drop of $3 \%$ aqueous solution of hydrogen peroxide observed for the production of gas bubbles by catalase-positive isolates. The oxacillin resistant, mannitol fermenter, Gram-positive cocci in bunch showing positive catalase activity were considered as MRSA isolates. Further these positive isolates were confirmed by $S$. aureus-specific polymerase chain reaction (PCR) targeting 16S rRNA using the primer sequence as F-5' GTAGGTGGCCAAGCGTTATCC 3' and R-5' CGCACATCAGCGTCAG 3' [13]. The PCR was performed with 1X PCR buffer ( $\mathrm{pH}-8.3 ; 15 \mathrm{mM} \mathrm{MgCl}$ ), $5 \mathrm{mM}$ of deoxynucleotide triphosphates (dNTPs), 20 pmol of forward and reverse primers, 1 U Taq DNA polymerase, $2 \mu \mathrm{l}$ of DNA template per reaction and final volume was adjusted to $25 \mu \mathrm{l}$ with nuclease free water. The cycling conditions were optimized with initial denaturation at $94^{\circ} \mathrm{C}$ for $5 \mathrm{~min}$, followed by 35 cycles of denaturation at $94^{\circ} \mathrm{C}$ for $1 \mathrm{~min}$, annealing at $50^{\circ} \mathrm{C}$ for $1 \mathrm{~min}$, and extension at $72^{\circ} \mathrm{C}$ for $1 \mathrm{~min}$ with the final extension at $72^{\circ} \mathrm{C}$ for $10 \mathrm{~min}$.

The confirmed isolates of MRSA were further screened for the presence of $m e c A$ gene by PCR. The PCR assay was standardized for amplification of mecA gene by forward (5' GTA GAA ATG ACT GAA CGT CCGATAA 3') and reverse (5'CCAATTCC ACATTGT TTCG GTC TAA 3') primer [14] with some modifications. The PCR reaction mixture was prepared in $25 \mu \mathrm{l}$ reaction volume each containing $2.5 \mu 1 \times 10$ PCR buffer (pH-8.3; $15 \mathrm{mM} \mathrm{MgCl}$ ), $0.5 \mu \mathrm{l}$ of dNTP mixture (10 $\mathrm{mM}$ each), $2 \mu \mathrm{l}(10 \mathrm{pmol} / \mu \mathrm{l})$ of forward and reverse primers, $1 \mu 1$ ( 1 unit) Taq DNA polymerase, $5 \mu 1$ of bacterial lysate and final volume was adjusted with nuclease free water. The bacterial lysate was prepared by boiling and snap chilling [15] from overnight grown culture in TSB. The cycling conditions used were initial denaturation at $94^{\circ} \mathrm{C}$ for $5 \mathrm{~min}$, followed by 32 cycles of denaturation at $94^{\circ} \mathrm{C}$ for $1 \mathrm{~min}$, annealing at $56^{\circ} \mathrm{C}$ for $1 \mathrm{~min}$ and extension at $72^{\circ} \mathrm{C}$ for $1 \mathrm{~min}$ with the final extension phase at $72^{\circ} \mathrm{C}$ for $10 \mathrm{~min}$.

The amplified products were analyzed by agarose gel electrophoresis using 1.5\% LE agarose (Hi media, India) on tris acetate-ethylenediaminetetraacetic acid buffer containing ethidium bromide $(0.5 \mu \mathrm{g} / \mathrm{ml})$. Gels were visualized and photographed under gel documentation system (Biorad).
Antibiogram study of MRSA isolates was performed by disc diffusion method [16] using a group of antibiotics, viz., ciprofloxacin (5 $\mu \mathrm{g})$, amoxiclav $(30 \mu \mathrm{g})$, ofloxacin $(5 \mu \mathrm{g})$, amikacin $(30 \mu \mathrm{g})$, cefoxitin $(30 \mu \mathrm{g})$, ceftriaxone $(30 \mu \mathrm{g})$, chloramphenicol $(30 \mu \mathrm{g})$, clindamycin $(2 \mu \mathrm{g})$, penicillin $(10 \mu \mathrm{g})$, gentamicin $(10 \mu \mathrm{g})$, oxytetracycline $(30 \mu \mathrm{g})$, tetracycline $(30 \mu \mathrm{g})$, cotrimoxazole $(25 \mu \mathrm{g})$, and vancomycin $(30 \mu \mathrm{g})$. The interpretation of results was made according to CLSI guidelines [9].

\section{Results}

A total of 136 nasal and skin swab samples were processed in this study. The prevalence of MRSA, out of 136 nasal and skin swab samples studied, is shown in Table-1. 22 and 31 MRSA isolates were collected from cattle and buffalo respectively, based on biochemical confirmation and amplification of S. aureus-specific 16S rRNA (228 bp) gene (Table1). The prevalence of MRSA was $28.57 \%$ and $34.28 \%$ in cattle nasal and skin swabs, respectively, with an overall prevalence of $31.43 \%$ among cattle, whereas buffalo nasal and skin showed $54.55 \%$ and $39.4 \%$, respectively, with an overall prevalence of $46.9 \%$ MRSA among buffalo (Table-1).

PCR amplification of the mecA gene has been used for specific and rapid identification of MRSA among the oxacillin-resistant $S$. aureus isolates. The test detected mecA gene (Figure-1) in 58.06\% MRSA isolates from buffalo, whereas only $36.4 \%$ of MRSA isolates from cattle were found positive for mecA gene. The frequency of detection of mecA gene in cattle nostril and skin swab was $50 \%$ and $25 \%$, respectively, wherein it was $50 \%$ and $69.23 \%$ in buffalo nostril and skin swab, respectively (Table-1).

Antimicrobial susceptibility test was performed for all the 53 MRSA isolates of cattle and buffalo origin. It revealed that $88 \%$ MRSA isolates of cattle origin, having $m e c \mathrm{~A}$ gene were resistant to penicillin and oxytetracycline, whereas $75 \%$ were resistant to cefoxitin followed by cotrimoxazole (62\%) and amoxyclav $(50 \%)$ whereas no resistance was observed against ciprofloxacin, amikacin, chloramphenicol, gentamicin, and vancomycin (Figure-2a). Similar pattern of

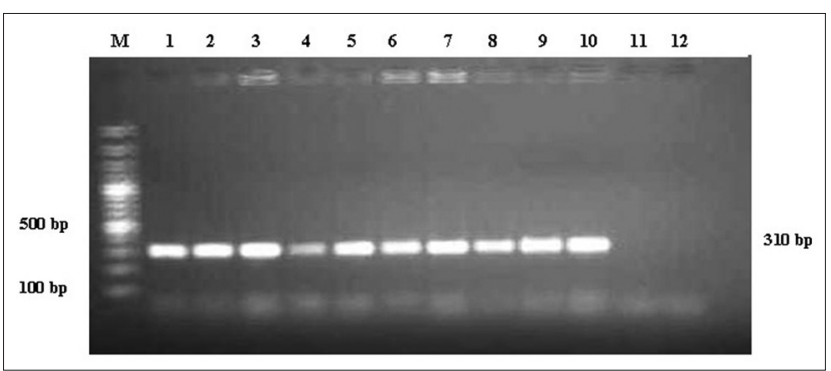

Figure-1: Polymerase chain reaction amplification of mecA gene of methicillin resistant Staphylococcus aureus. M: Gene ruler 100 bp plus DNA ladder, L1: Positive control, L2-L10: Positive amplicons of methicillin resistant Staphylococcus aureus, L11: Isolate with no amplicon, L12: Negative control. 
Table-1: Prevalence of MRSA among cattle and buffalo.

\begin{tabular}{lccc}
\hline Sample type & $\mathbf{n = 1 3 6}$ & $\begin{array}{c}\text { \% MRSA detected by mannitol fermentation and } \\
\text { 16S rRNA amplification (n) }\end{array}$ & $\begin{array}{c}\text { \% mecA positive MRSA (n) } \\
\text { Cattle }\end{array}$ \\
$\quad$ & & $28.57(10)$ & $50.00(05)$ \\
Nose swab & 35 & $34.28(12)$ & $25.00(03)$ \\
Skin swab & 35 & $31.43(22)$ & $36.36(08)$ \\
Sub total & 70 & $54.55(18)$ & $50.00(09)$ \\
Buffalo & & $39.39(13)$ & $69.23(09)$ \\
Nose swab & 33 & $46.97(31)$ & $58.06(18)$ \\
Skin swab & 33 & & \\
Sub total & 66 & & \\
\hline
\end{tabular}

$\mathrm{N}$ : Total number of samples; $\mathrm{n}$ : number of samples in each column. MRSA=Methicillin resistant Staphylococcus aureus



Figure-2: Antimicrobial susceptibility test result for methicillin resistant Staphylococcus aureus from cattle (a) and buffalo (b). CIP: Ciprofloxacin, AMC: Amoxiclav, OF: Ofloxacin, AK: Amikacin, CX: Cefoxitin, CTR: Ceftriaxone, C: Chloramphenicol, CD: Clindamycin, P: Penicillin, GEN: Gentamicin, O: Oxytetracycline, TE: Tetracycline, COT: Cotrimoxazole and VA: Vancomycin.

antimicrobial resistance for penicillin, cotrimoxazole, cefoxitin, ciprofloxacin, amikacin and chloramphenicol was observed in mecA positive isolates of buffalo origin. The finding also revealed resistance against vancomycin, in $16.7 \%$ of MRSA isolates from buffalo (Figure-2b).

\section{Discussion}

The findings of this study revealed that prevalence of MRSA in buffalo (46.9\%) was higher in comparison to cattle $(31.43 \%)$ which corroborate with earlier findings on isolation of MRSA from nasal or skin samples of cattle $[17,18]$. However, no reports are available on isolation of MRSA from buffalo nasal and skin samples. Although low prevalence $(0.7 \%)$ of MRSA harboring in nose and skin of pigs have been reported from Thailand [19]. The difference in the sample size and geographical variations may be the causes of this discrepancy in the prevalence of MRSA. The presence of mecA gene which encodes a modified penicillin-binding protein (PBP), i.e., PBP2a is a useful molecular marker of $\beta$-lactam resistance in Staphylococci $[20,21]$. Hence, PCR amplification of the mecA gene have been used in the present study for specific identification of MRSA among the oxacillin-resistant $S$. aureus isolates [22,23]. The study revealed a total $36.36 \%$ and $58.06 \%$ of MRSA isolates carrying mecA gene from cattle and buffalo, respectively. Similar findings of mecA gene detection in cattle nasal carriage have been reported by Alzohairy [24]. In contrast, a lower prevalence of
$0.3 \%$ and $1 \%$ of MRSA has been reported in farm cattle and calves [25]. The lower percentage of mecA gene amplification by PCR among MRSA isolates in the current study may be due to penicillinase activity of $S$. aureus [26] or due to the presence of new mecA gene homologue, mecALGA251 called mecC gene in the isolates [27] which could be responsible for MRSA isolates.

The antibiogram study revealed resistance to penicillin and oxytetracycline (88\%) in MRSA isolates of cattle, whereas $75 \%$ were resistant to cefoxitin, (62\%) cotrimoxazole and (50\%) amoxyclav . No resistance was observed against ciprofloxacin, amikacin, chloramphenicol, gentamicin and vancomycin. Similar pattern of antimicrobial resistance for penicillin, cotrimoxazole, cefoxitin, ciprofloxacin, amikacin, and chloramphenicol was observed in mecA positive isolates of buffalo origin. It revealed that $88 \%$ MRSA isolates of cattle origin, having mecA gene were resistant to penicillin and oxytetracycline whereas $75 \%$ were resistant to cefoxitin followed by cotrimoxazole $(62 \%)$ and amoxyclav (50\%) whereas no resistance was observed against ciprofloxacin, amikacin, chloramphenicol, gentamicin, and vancomycin (Figure-2a). This antimicrobial resistance pattern was found similar to that of buffalo isolates. The finding also revealed resistance against vancomycin, in $16.7 \%$ of MRSA isolates from buffalo. Kumar et al. [8], Vishnupriya et al. [9], and Chandrasekaran et al. [10] have the similar findings of antimicrobial resistance against a group of antibiotics in MRSA. 
The current finding also revealed resistance against vancomycin, in $16.7 \%$ of MRSA isolates from buffalo which is considered as the drug of choice for treatment of MRSA infections [28]. Although a low level of resistance against vancomycin has been reported earlier [28], only a few intermediate susceptibility but no resistance was against vancomycin has been reported [11]. Hence, the isolation of vancomycin-resistant $S$. aureus (VRSA) in the current study indicates the emergence of VRSA in animal population which may have a serious implication in human infection.

\section{Conclusion}

The present finding concludes that cattle and buffalo may act as a potential carrier of MRSA which may act as a risk factor for human's infection who are in direct contact with live animals [29].

\section{Authors' Contributions}

PK was the mentor and project leader. AK and Anjay were responsible for project design and performed most of the work. PK and MK were responsible for experimental work and support. All authors read and approved the final manuscript.

\section{Acknowledgments}

Authors wish to acknowledge the Honbl'e Vice Chancellor, BAU, Sabour and Dean BVC, Patna, for providing all the necessary funds (BAU Communication No. 175/2016), technical support and infrastructure for conducting the study.

\section{Competing Interests} interests.

The authors declare that they have no competing

\section{References}

1. Vanderhaeghen, W., Herman, K., Haesebrouck, F. and Butaye, P. (2010) Methicillin resistant Staphylococcus aureus (MRSA) in food production animals. Epdemiol. Infect., 138: 606-625.

2. Wertheim, H.F.L., Lelles, D.C., Vos, M.C., van Leeuwen, W., van Belkum, A., Verbrugh, H.A. and Nouwen, J.L. (2005) The role of nasal carriage in Staphylococcus aureus infections. Lancet Infect. Dis., 5: 751-762.

3. Sangappa, S. and Thiagarajan, P. (2012) Methicillin resistant S. Aureus: Resistance gene and their regulation. Int. J. Pharm. Pharm. Sci., 4: 658-667.

4. Anon. (2001) European Antimicrobial Resistance Surveillance System (EARSS). Annual Report. National Institute of Public Health and the Environment, Bilthoven.

5. Rohrer, S., Bischoff, M., Rossi, J. and Berger-Bachi, B. (2003) Mechanisms of methicillin resistance. In: Fluit, A.C. and Schmitz, F.J., editors. MRSA: Current Perspectives. Caister Academic Press, Norfolk, England. p31-53.

6. Ito, T., Katayama, Y., Asada, K., Mori, N., Tsutsumimoto, K., Tiensasitorn, C. and Hiramatsu, K. (2001) Structural comparison of three types of staphylococcal cassette chromosome mec integrated in the chromosome in methicillin-resistant Staphylococcus aureus. Antimicrob. Agents Chemother, 45: 1323-1336.

7. Ma, X.X., Ito, T., Tiensasitorn, C., Jamklang, M., Chongtrakool, P., Vavra, S.B., Daum, R.S. and Hiramatsu, K.
(2002) Novel type of staphylococcal cassette chromosome mec identified in community-acquired methicillin resistant Staphylococcus aureus strains. Antimicrob. Agents Chemother., 46: 1147-1152.

8. Kumar, R., Yadav, B.R. and Singh, R.S. (2011) Antibiotic resistance and pathogenicity factors in Staphylococcus aureus isolated from mastitic sahiwal cattle. J. Biosci., 36: 175-188.

9. Vishnupriya, S., Antony, P.X., Mukhopadhyay, H.K., Pillai, R.M., Thanislass, J., Srinivas, V.M. and Kumar, S.R. (2014) Methicillin resistant staphylococci associated with bovine mastitis and their zoonotic importance. Vet. World, 7: 422-427.

10. Chandrasekaran, D., Venkatesan, P., Tirumurugaan, K.G., Gowri, B., Subapriya, S. and Thirunavukkarasu, S. (2014) Sub-acute mastitis associated with methicillin resistant Staphylococcus aureus in a cow: A case report. J. Adv. Vet. Anim. Res., 1: 235-237.

11. Sharma, L., Verma, A.K., Kumar, A., Rahat, A., Neha. and Nigam, R. (2015) Incidence and pattern of antibiotic resistance of Staphylococcus aureus isolated from clinical and subclinical mastitis in cattle and buffaloes. Asia. J. Anim. Sci., 9: 100109.

12. Agarwal, R.K., Bhilegaonkar, K.N., Singh, D.K., Kumar, A. and Rathore, R.S. (2003) In: Laboratory Manual for the Isolation and Identification of Food Borne Pathogens. $1^{\text {st }}$ ed. IVRI, Izzatnagar. p38-39.

13. Monday, S.R. and Bohach, G.A. (1999) Use of multiplex PCR to detect classical and newly described pathogenic toxin gene in staphylococcal isolates. J. Clin. Microbiol., 37: 3411-3414

14. Braoios, A., Fluminhan, J.A. and Pizzolitto, A.C. (2009) Multiplex PCR use for Staphylococcus aureus identification and oxacillin and mupirocin resistance evaluation. $J$. Basic Appl. Pharm. Sci., 30: 303-307.

15. Kaushik, P., Anjay, Kumari, S., Bharti, S.K. and Dayal, S. (2014) Isolation and prevalence of Salmonella from chicken meat and cattle milk collected from local 30: Markets of Patna, India. Vet. World, 7: 62-65.

16. Wayne, P.A. (2002) Performance Standards of Antimicrobial Susceptibility: National Committee for Clinical Laboratory Standards (NCCLS). NCCLS Approved Standards. p100-159.

17. Garipcin, M. and Seker, E. (2015) Nasal carriage of methicillin-resistant Staphylococcus aureus in cattle and farm workers in Turkey. Vet. Arch., 85: 117-129.

18. Inegol, E. and Turkyilmaz, S. (2012) Determination of SCC mec types in methicillin resistant staphylococci isolated from cows and farm workers. Ankara. Univ. Vet. Fak. Derg., 59: 89-93.

19. Patchnee, P., Tadee, P., Arjkumpa, O., Love, D., Chanachai, K., Alter, T., Hinjoy, S. and Tharavichitkul, P. (2014) Occurrence and characterization of livestock associated methicillin resistant Staphylococcus aureus in pig industries of Northern Thailand. J. Vet. Sci., 15: 529-536.

20. Mulligan, M.E., Murray, K.A., Standiford, H.C., John, J.F., Kauffmann, C.A. and Yu, V.L. (1993) Methicillin-resistant Staphylococcus aureus: A consensus review of the microbiology, pathogenesis, and epidemiology with implications for prevention and management. Ame. J. Med., 94:313-328.

21. Pinho, M.G., Filipe, S.R., de Lencastre, H. and Tomasz, A. (2001) Complementation of the essential peptidoglycan trans-peptidase function of penicillin-binding protein 2 (PBP2) by the drug resistance protein PBP2A in Staphylococcus aureus. J. Bacteriol., 183: 6525-6531.

22. Choi, S.M., Kim, S., Kim, C., Lee, D., Choi, J., Yoo, J., Kang, J., Shin, W. and Kang, W. (2003) Multiplex PCR for the detection of genes encoding aminoglycoside modifying enzymes and methicillin resistance among Staphylococcus species. J. Korean Med. Sci., 18: 631-636.

23. Kalhor, H., Shariati, L., Validi, M., Tabatabaiefar, M.A. and Nafisi, M.R. (2012) Comparison of agar screen and 
duplex-PCR methods in determination of methicillin-resistant Staphylococcus aureus (MRSA) strains isolated from nasal carriage. Afr. J. Microbiol. Res., 6: 3722-3726.

24. Alzohairy, M.A. (2011) Colonization and antibiotic susceptibility pattern of methicillin resistance Staphylococcus aureus (MRSA) among farm animals in Saudi Arabia. $J$. Bacteriol. Res., 3: 63-68.

25. Huber, H., Koller, S., Giezendanner, N., Stephan, R. and Zweifel, C. (2010) Prevalence and characteristics of methicillin-resistant Staphylococcus aureus in humans in contact with farm animals, in livestock, and in food of animal origin, Switzerland, 2009. Eur. Surv., 15: 7-10.

26. Lowy, F.D. (1998) Staphylococcus aureus infections. N. Engl. J. Med., 339: 520-532.
27. Peterson, G.K., Larsen, J., Harrison, E.M., Larsen, A.R., Morgan, F.J., Peacock, S.J., Parkhill, J., Zadoks, R.N. and Holmes, M.A. (2012) First detection of livestock-associated meticillin resistant Staphylococcus aureus CC398 in bulk tank milk in the United Kingdom. Eur. Surv., 17: 20337.

28. Assadullah, S., Kakru, D.K., Thoker, M.A., Bhat, F.A., Hussain, W. and Shah, A. (2003) Emergence of low level vancomycin resistance in MRSA. Indian J. Med. Microbiol., 21: 196-198.

29. Beninati, C., Reich, F., Muscolino, D., Giarratana, F., Panebianco, A., Klein, G. and Atanassova, V. (2015) ESBLproducing bacteria and MRSA isolated from poultry and Turkey products imported from Italy. Czech. J. Food Sci., 33: 97-102.

$* * * * * * * *$ 